\title{
Museos Comunitarios como una Estrategia de Comunicación Intercultural Ancestral en Nueva Guinea, RACCS
}

\author{
Community Museums as an Ancestral Intercultural \\ Communication Strategy in Nueva Guinea, RACCS
}

Greyci Sobalbarro Pao ${ }^{1}$

Yuri Hamed Zapata Webb ${ }^{2}$

\section{Resumen}

La cosecha confirma que el Museo Comunitario "Los Ranchitos", es una estrategia de comunicación intercultural ancestral en Nueva Guinea, que aporta notablemente a la herencia cultural y a la revitalización de la identidad colectiva del pueblo mestizo. También, fortalece la Red Nicaragüense de Museos Comunitarios. Consistentemente, el estudio permite documentar el trabajo que desde el territorio se emprende para el rescate del patrimonio cultural. Este es un estudio cualitativo, guiado por "el camino de Creación, Recreación de Conocimientos, Saberes y Prácticas de URACCAN", en una estrategia que aseguró el involucramiento directo de la comunidad articulada desde la red nicaragüense de museos comunitarios y el comité amigos del museo. El estudio destaca que si bien estos espacios son un instrumento para la protección y preservación del patrimonio cultural y la identidad del pueblo mestizo de Nueva Guinea, hace falta mayor divulgación, creación de conciencia y cuido en su calidad de sitios ancestrales.

Palabras clave: Museos comunitarios; sitios ancestrales; Red Nicaragüense Museos Comunitarios; Comité Amigos del Museo; preservación; patrimonio cultural.

\section{Abstract}

The harvest confirms that "Los Ranchitos" Community Museum is an ancestral intercultural communication strategy in Nueva Guinea, which contributes significantly to the cultural heritage and revitalization of the mestizo people collective identity. Also, it strengthens the Nicaraguan Network of Community Museums. Consistently, the study allows documenting the work that is undertaken from the territory for rescuing of the cultural heritage. This is a qualitative study, guided by "the path of Creation, Recreation of Knowledge, Knowings and Practices of URACCAN", in a strategy that ensured the direct involvement of the community articulated from the Nicaraguan network

1 Máster en Comunicación Intercultural con Enfoque de Género. Responsable del Museo Comunitario Los Ranchitos de URACCANNueva Guinea, Costa Caribe de Nicaragua. Correo Electrónico: greysipauth@yahoo.es; ORCID: https:/orcid.org/o0oo-0001-8275-687x

2 Candidato Doctoral en Educación Superior, Máster en Estudios Interdisciplinarios, Vicerrector General de URACCAN. Correo Electrónico: yuri.zapata@uraccan.edu.ni; ORCID: https://orcid.org/oooo-0o02-4843

Recibido: 21/05/2019 Aprobado: 29/07/2019 
of community museums and the museum friends committee. The study highlights that although these spaces are an instrument for the protection and preservation of the cultural heritage and identity of the mestizo people from Nueva Guinea, there is a need for greater dissemination, awareness raising and care in their quality as ancestral sites.

Keywords: Community Museums; ancestral sites; Nicaraguan Community Museums Network; Museum Friends Committee; preservation; cultural heritage.

\section{Introducción}

Los Museos Comunitarios como una estrategia de comunicación intercultural ancestral en Nueva Guinea, es un proceso de cultivo y crianza, cuyo objetivo principal radica en constatar que estos movimientos comunitarios, son una herramienta para ejercer la comunicación intercultural de los pueblos originarios de la región.

El estudio es el resultado del interés que tengo de rescatar los esfuerzos que se han venido consolidando a la luz de la Red de Museos Comunitarios de las Américas y la Red de Museos Comunitarios de Nicaragua, con la plena convicción de caminar en unidad en la recuperación del patrimonio cultural de los pueblos.

Este trabajo es de gran relevancia dado que analiza los museos comunitarios desde una perspectiva comunicacional y reivindica el compromiso asumido por la universidad URACCAN en la construcción y fortalecimiento de ciudadanías interculturales, el respeto a la cultura y el buen vivir de los pueblos de la región. Por ende, es un estudio que se enfoca en el gran valor histórico cultural que poseen los museos comunitarios en el rescate de la identidad y la protección del patrimonio arqueológico existente en Nueva Guinea y el país.

Su valor investigativo radica en ser uno de los primeros estudios con la iniciativa de comprobación comunicacional, por tal razón se procede a la indagación de procesos comunitarios que se producen en esta parte del territorio nacional.

Relevante es profundizar en la importancia de los sitios arqueológicos de Nueva Guinea, como resguardo del patrimonio cultural y legado ancestral de nuestros pueblos indígenas que habitaron estas tierras en tiempos pasados, y donde el Museo Comunitario Los Ranchitos centra la investigación, divulgación y promoción a través de su museología, talleres, giras de campo y charlas.

El documentar la importancia que el museo presenta en Nueva Guinea, como el punto céntrico hacia el vivir la memoria del pueblo mestizo, la idiosincrasia y el respeto que se le debe de dar a los sitios ancestrales, es el resultado que se quiere ejemplificar en este trabajo de cosecha que se está realizando. 
El Museo Comunitario Los Ranchitos a través de su trabajo comunitario y la ampliación de la red de museos a nivel nacional ha aportado a la comunicación intercultural dándole voz a las comunidades de contar su propia vida, historia y cultura.

Otro de los aspectos importantes, es el de vivenciar cómo el museo comunitario colabora al fortalecimiento y la práctica de la identidad de los pueblos con la representación de sus tradiciones, cultura, historias, simbología ancestral que tiene el pueblo mestizo asentado en el área geográfica de Nueva Guinea.

\section{Revisión de literatura}

\section{Revisión de Sabidurías y Conocimientos para el Cultivo y Crianza}

\section{Regiones Autónomas de Nicaragua:}

Las Regiones Autónomas de la Costa Caribe de Nicaragua, posee riquezas culturales en diversa índole, profundizando en el tipo arqueológico, en este sentido hace pensar que estos territorios estuvieron poblados desde muchísimo tiempo confirmado por algunos investigadores y se puede decir que estuvo poblado desde mucho antes de la llegada de los europeos en el actual territorio nicaragüense.

(Zapata, 2007) nos narra;

"La composición cultural y social de las Regiones Autónomas ha estado precedida por procesos de migración de culturas milenarias a lo largo de varios siglos de nuestra era. Estos diversos procesos migratorios son los que posibilitaron tener una diversidad étnico-cultural asentada en la región, cuando llegaron los colonizadores europeos a estas tierras, encontraron esta diversidad, por otro lado, los procesos de colonización y neocolonización permitieron el asentamiento de otras identidades étnico culturales, lo que terminó de enriquecer la amalgama cultural de esta área geográfica de Nicaragua". (p.15).

El territorio nicaragüense está dividido por dos regiones autónomas; Región Autónoma Costa Caribe Sur (RACCS) y Región autónoma de la Costa Caribe Norte (RACCN), prevaleciendo los grupos étnicos ancestrales en estos dos territorios, actualmente clasificados de esta manera; Mayangnas, Ramas, miskitus, Garifonas, Creoles y Mestizos. 


\section{Aspectos Generales de Nueva Guinea (Arqueología)}

Nueva Guinea es una de las ciudades más jóvenes de la Región Autónoma Costa Caribe Sur, ubicado al sureste de Nicaragua, con las coordenadas $11^{\circ} 43^{\prime \prime}$ latitud norte y $84^{\circ}$ 30" longitud oeste.

La ciudad de Nueva Guinea posee evidencias culturales de diversa índole, se puede comprobar que existen evidencias de tipos arqueológicas, por ello hace pensar que este territorio estuvo poblado desde hace mucho tiempo antes de la colonización.

En este sentido, (López \& Ramos, 2012) nos explica;

El territorio de Nueva Guinea se encuentra en la frontera de las zonas de la Gran Nicoya y la Costa Caribe de Nicaragua con influencia del sector sur de la subregión de la Gran Nicoya. Puede afirmarse que existe un influjo cultural de los pueblos del Caribe y los de la región del Pacífico, y entre ambas áreas culturales se localiza una franja donde ambas comparten características esenciales. (p.18)

En Nueva Guinea se aprecian procesos sociales, económicos y productivos que influyen positiva y negativamente. Uno de ellos es en el ámbito arqueológico que por la falta de cuido es difícil la recopilación de datos históricos-culturales que nuestros ancestros han dejado, en este sentir se ve la necesidad de priorizar estos aspectos históricos, para el fortalecimiento del museo que nos sirve para el resguardo y cuido del patrimonio de la zona.

En estudios sobre prospecciones arqueológicas documentadas por:

(Ruiz, Vizcaya, \& García, 2003) se ha podido observar las profundas afectaciones que se están dando a través de las profanaciones de huaqueros, actividades agrícolas de tumba, rosa y quema, actividades ganaderas que a su vez han destruido montículos (tumbas, cementerios indígenas) y otras piezas arqueológicas, que nos dan idea de la vida comunitaria ancestral, se encuentran en manos de particulares.

\section{Comunicación Intercultural}

Los museos comunitarios aparte de ser una herramienta para el fortalecimiento de nuestras comunidades, promulgan una estrecha comunicación intercultural en el rescate y protección de las culturas de los pueblos, a través del ejercicio de las asambleas comunitarias donde la población tiene el vos y voto de las tomas de decisiones. 
(Rodrigo Alcina, 1999) dice;

Pero no hay que conocer solo otras culturas, sino que la comunicación intercultural implica también una toma de conciencia de la propia cultura (aquí comenzaría la cita). "En muchas ocasiones nuestras comunicaciones están llenas de valores que transmitimos sin ser apenas conscientes de ello. La comunicación intercultural no solo supone comunicarse con otras culturas sino también hacer el esfuerzo de repensar la propia cultura". (p.6)

Corroborar que los museos comunitarios aparte de ser unos espacios del resguardo del patrimonio cultural, también son agentes del diálogo intercultural como una nueva forma de incorporar la comprensión de la historia y arqueología, encontrada para lograr interpretar a las culturas a través del respeto y reconocimiento de nuestros ancestros.

Para la ciudad de Nueva Guinea el museo comunitario, representa el sentir de la comunidad. A través de las distintas actividades presentadas se reflejan las vivencias propias de los personajes de este pueblo, su idiosincrasia, su cultura, historia y mucho más.

\section{Museos comunitarios como fortalecimiento de la identidad de los pueblos}

\section{¿Qué es un museo comunitario?}

Los museos comunitarios son un espacio para contribuir a la lucha de las comunidades, como procesos de gestión ante situaciones que viven las comunidades, son una manera de comunicar las problemáticas que están siendo censuradas y no escuchadas a través del consenso colectivo y la memoria de los pueblos.

(Morales \& Cuathemoc, 2009) p. 15 expresan:

"Un museo comunitario es un espacio donde los integrantes de la comunidad construyen un autoconocimiento colectivo, propiciando la reflexión, la crítica y la creativa, fortalece la identidad, porque legitima la historia y los valores propios, proyectando la forma de vida de la comunidad hacia adentro y hacia fuera de ella. Fortalece la memoria que alimenta sus aspiraciones de futuro".

(Gamboggi \& Melville) comentan:

Es por medio de las memorias (sean de olvidos o de recuerdos) que cobra una significación especial de la pertenencia a una comunidad, la memoria colectiva expresa los valores culturales del grupo, una vez que la memoria es constituida por medio de una selección de hechos memorables, dignos del recuerdo por parte de ese grupo. A 
partir de esa selección podemos ver que criterios el grupo utilizara para seleccionar cuáles memorias harán parte de la historia identitaria del grupo (p.4).

Los museos comunitarios son la viva voz del pueblo, los que toman la decisión sobre que se debe de exponer en tal espacio, y los que fomentan el rescate del patrimonio cultural, analizan sus propias necesidades para organizarse y buscar estrategias para las posibles soluciones de sus comunidades.

Los museos comunitarios responden a necesidades y derechos que tienen las comunidades, una forma de subsistir a través de los tiempos e importante recalcar que es creado por la misma comunidad.

\section{¿Para qué sirven los Museos Comunitarios?}

Seguir los procesos comunitarios y que ellos se sientan identificados es cuestión de trabajo, organizar, concientizar y divulgar, por eso los museos comunitarios van más allá de un local muerto de exhibición de objetos antiguos, son medios de comunicación para capacitar, apropiar, involucrar a nuestras comunidades indígenas y grupos étnicos.

(Morales, Camarena, \& Valeriano, 1994) sintetiza que;

El museo se vuelve un punto de partida para generar múltiples iniciativas culturales. Su permanencia favorece el desarrollo continuo de nuevas opciones, que puedan proyectarse en la investigación, la capacitación y la revitalización cultural a través de la danza, música y teatro. (p.11).

\section{Red de Museos Comunitarios de las Américas}

La Red de Museos Comunitarios de las Américas, está conformada a nivel internacional y desde el año 2000 se consolidó con más de diez países con la intención de generar mejores oportunidades para sus comunidades, esta red está coordinada por la primera red “Unión de Museos Comunitarios de Oaxaca, México, la cual nació en el año 1991.

Esta unión de iniciativas de base comunitaria, está integrada por comunidades comprometidas en la defensa de su forma de vida y su cultura a través de los museos comunitarios, las cuales se vinculan por diversas formas de representación de sus órganos colectivos. (López \& Sobalbarro, 2016)

\section{Red de Museos Comunitarios de Nicaragua}

Es una organización que nació en el año 2013, con el fin de fortalecer el resguardo del patrimonio histórico cultural de Nicaragua y recopilar la memoria colectiva de los pueblos, es un espacio de organización regional y nacional que garantiza que la 
cultura de los pueblos no se desaparezca, esta organización con el acompañamiento de la Universidad de las Regiones Autónomas de la Costa Caribe Nicaragüense y la Red de Museos Comunitarios de las Américas, han abierto espacios para la constante capacitación de los museos comunitarios de nuestro país.

La Red Nicaragüense de Museos Comunitarios nace como una iniciativa de las comunidades y del interés en común por preservar y conservar el patrimonio cultural propio. Desde 1996 la Unión de Museos Comunitarios de Oaxaca establece contactos con la rectoría de Universidad de las Regiones Autónomas de la Costa Caribe Nicaragüense (URACCAN); a partir de esto la universidad participa en el primer encuentro internacional de Museos Comunitarios de América en el año 200o. (López \& Sobalbarro, 2016).

\section{Museo Comunitario Los Ranchitos y su origen en Nueva Guinea}

Surgió por el involucramiento de la población para su creación, con asesoramiento de la Universidad URACCAN y apoyo de estudiantes de la Carrera de Ciencias de la Educación y Humanidades con mención en Historia.

Quienes realizaron el proceso de investigación sobre el potencial arqueológico en las colonias del sur y suroeste del municipio en el marco de la asignatura de arqueología, los resultados mostraron la suma importante de piezas arqueológicas para la sociedad nicaragüense. Desde entonces el gobierno municipal, la policía nacional, autoridades de instituciones municipales, pobladores y estudiantes tomaron la decisión de conservarlo y establecer el museo en las instalaciones de la universidad URACCAN. Lo anterior contó con el apoyo del BID y el departamento de patrimonio cultural de Nicaragua.

El Museo Comunitario "Los Ranchitos" desde su fundación siempre ha estado funcionando en el local de la universidad y la comunidad universitaria se incorpora en las acciones de fortalecimiento del mismo y las actividades promovidas por él.

Por otra parte, Nueva Guinea, con tan sólo 53 años de existencia, tiene la necesidad de ampliar la historia del municipio y sus colonias, recuperar tradiciones, creencias, mitos, leyendas, cuentos, religión, cultura y objetos antiguos e históricos que utilizaron los primeros pobladores en su llegada en los años 1960 y desde estas iniciativas se convive con las comunidades para seguir uniendo esfuerzos para realizar acciones en pro del rescate de la memoria colectiva de los pueblos. 


\section{Materiales y métodos}

\section{Selección de procedimientos de cultivos y crianza}

\section{Caminos y formas del CCRISAC}

El reto en este cultivo y crianza es experimentar, convivir, relacionarme con mis hermanos, mi gente, mi comunidad, para solucionar mi problema y comprobar que los museos comunitarios son una estrategia de comunicación ancestral. Este aprendizaje se llevó a cabo en la ciudad de Nueva Guinea en la Región Autónoma del Caribe Sur. Con una extensión territorial de 2 mil 774 kilómetros cuadrados y una población de 136 mil 349 habitantes, el lugar donde se ubicará el estudio estará determinado con actores que ayudaran en el caminar con la información detallada desde el ámbito urbano y comunitario, redes que han estado trabajando en el quehacer de los museos comunitarios y comité amigos del museo.

Con este esfuerzo comunitario se intenta documentar las vivencias que se han realizado desde los museos comunitarios y comprobar que estos son espacios de memoria colectiva, organizativa y una forma estrategia comunicacional ancestral, comunitaria y social. Este fruto será para comprobar que los museos comunitarios trabajan para el fortalecimiento comunitario, vinculando procesos de apropiación cultural en el que puedan fortalecer su identidad, autonomía y revitalización cultural.

El universo para el estudio se realizó con líderes comunitarios participantes de los talleres de museos comunitarios, con comité amigos del museo que son parte de la población de ciudad Nueva Guinea, Red Nicaragüense de Museos Comunitarios, Red de Museos Comunitarios de las Américas. El avance de investigación es dirigido al presidente y facilitadores de la Red nicaragüense de museos comunitarios, Comité amigos del museo, Líderes comunitarios participantes de talleres de museos comunitarios.

\section{Resultados y discusión}

\section{Sistematización del CRISSAC}

Esta cosecha está dirigida desde el recinto universitario URACCAN Nueva Guinea, la Red Nicaragüense de Museos Comunitarios y el Comité Amigos del Museo con quienes se trabaja y articulan acciones desde el Museo Comunitario Los Ranchitos, entre 2003-2018. 


\section{Herencia cultural, ancestral e identitaria del pueblo mestizo de Nueva Guinea desde la representación del museo comunitario Los Ranchitos}

Los museos comunitarios hoy en día se han convertido en una de las principales herramientas de apropiación de la identidad de los pueblos, un trabajo conjunto que se ha fortalecido con la conformación de redes nacionales e internacionales. El Museo Comunitario Los Ranchitos ha sido un ente regulador para recuperar la memoria colectiva e identitaria de este pueblo mestizo, es el eslabón en la constitución de la red de museos comunitarios de Nicaragua; el primer museo creado en Nicaragua, como iniciativa de URACCAN, en coordinación con la Red de Museos Comunitarios de las Américas.

Nueva Guinea es una zona rica en espacios ancestrales, en sus colonias se evidencian hallazgos arqueológicos, esto posiblemente referencia dos posibles teorías sobre el origen primario de sus habitantes, la primera, se señala dentro de la Gran Nicoya, región histórica de Mesoamérica, la segunda, a la presencia de los grupos micro-chibchas, afirmación que se hace por la presencia de cerámica tanto del este como del norte de américa (López y Ramos, 2012).

El museo comunitario es un espacio en el que se recrea la historia del pueblo mestizo de Nueva Guinea. Se transmite el significado de los abuelos y abuelas contados a través de los objetos antiguos cargados de historias vividas y recordadas, desde una piedra de moler hasta una vasija de agua. Durante 5 años, he sido testigo de miradas de nostalgia, sorpresas, emociones y recuerdos cuando me entregan un objeto antiguo e histórico, es por eso que afirmo que los museos comunitarios son un espacio comunicativo que transciende desde lo ancestral hasta lo actual.

Haciendo un balance de cuál es la situación hoy de los museos, el planteamiento que hace (Martínez M. , 2018) es el siguiente:

"Actualmente la comunidad está más relacionada al Museo Comunitario, interesándose en exposiciones y actividades que se realizan, a donaciones de piezas, donde niños y adultos visitan el Museo con el propósito de conocer, aprender y obtener información".

Para la Red Nicaragüense de Museos Comunitarios:

"Los Museos fortalecen el apego de las comunidades a sus valores culturales, patrimoniales e históricos, acentuando con ello los elementos propios de la identidad comunitaria. Una de las experiencias vividas ha sido pertenecer a esta red en la que se construye un intercambio cultural, valorizando el rescate, promoción y resguardo de nuestra historia en donde la comunidad es la que cuenta su sentir, vivencia y cosmovisión de su desarrollo. 
El Museo Comunitario ha permitido que la comunidad, entienda una forma diferente de organizarse para rescatar, resguardar, valorizar y promocionar su historia y cultura, el museo ha sido visto como un proceso y un espacio que ha despertado el interés por contar la historia cotidiana del pueblo donde ellos pueden entrar en contacto con los rostros que pocas veces se encuentran integrados en las páginas de la historia escrita”. (Vargas M. , 2018)

Para (Martínez M. , 2018): una estrategia interesante para transmitir la historia, es a través de la preservación de objetos históricos y arqueológicos donde las nuevas generaciones puedan conservar su identidad nicaragüense en cada una de ella”. Desde la lógica de los actores claves entrevistados las colonias y comunidades de Nueva Guinea son un potencial para el desarrollo cultural, comunitario y eco turístico, y a través de la difusión de las acciones del museo fortalecen la identidad comunitaria.

En este proceso de enseñanza, no se puede obviar los acontecimientos presentados que existen en este territorio mestizo, los hallazgos arqueológicos que se tienen que estudiar a profundidad para descubrir, comprender e interpretar a los pueblos que se asentaron en este territorio un legado ancestral que desde el museo comunitario Los Ranchitos se investiga para enriquecer la museología y rescate cultural de los ancestros.

\section{Contribución del museo comunitario Los Ranchitos a la construcción de la identidad colectiva del pueblo mestizo de Nueva Guinea}

El museo comunitario Los Ranchitos, es portador de un gran legado histórico-cultural, iniciando desde su exhibición en su sala museológica hasta en el trabajo de campo que realiza, hay cuatro componentes importantes en la que da el significado sobre como aporta al fortalecimiento de la identidad de la población mestiza de Nueva Guinea: Concientización comunitaria, Organización en redes comunitarias, nacionales e internacionales, Difusión y divulgación (ferias, charlas, talleres, visitas, giras de campo, tradiciones), Juegos Tradicionales.

\section{Aporte a la Comunicación Ancestral desde el Museo Comunitario Los Ranchitos:}

"Museos vivos" ó "museos comunitarios" como se les quiera llamar, al final son un instrumento para la afirmación de la identidad, tradición y cultura de cada pueblo. Son un medio de comunicación desde que se encuentra un símbolo ancestral (pieza arqueológica). Son historia, quehacer comunitario e idiosincrasia que se vuelve comunicación. Una ventana a la historial ancestral de los pueblos.

Los museos comunitarios nos permiten conocer, casi a viva voz, esos personajes del pueblo de tiempos ya pasados que tienen tantas vivencias que contar sobre nuestras tradiciones, cultura e historia. El proceso permite un proceso de comunicación 
constante y enriquecedor para el acervo patrimonial de nuestro pueblo. (Vargas M. , 2018).

\section{Sitios arqueológicos (sitios ancestrales):}

EL Museo Comunitario Los Ranchitos, cuenta con una variedad en metates, (piedras de moler), lítica (primer material utilizado por el hombre), y cerámica (barro). La colección ubicada en "Comarca Los Ranchitos", encarna formas de interpretación y de vida, comunicación y creación de los antepasados, su comercio, organización social, tradiciones, juegos, ceremonias, rituales, tecnología agrícola, y medicina tradicional.

El museo, al organizarse en sus comunidades también se involucra en conocer, comprender y trasmitir la cultura de los ancestros. A manera de ejemplo, el estudio de los petroglifos (arte rupestre) en comarca "La Letra" ayuda a comprender la manera que utilizaban la simbología para comunicarse. Con el estudio se logró constatar que el museo trabaja con sitios ancestrales de la zona que lamentablemente son vistos como un campo de investigación arqueológico, olvidándose de lo esencial: el respeto a estos espacios.

\section{Museo Comunitario y la Interculturalidad:}

La interculturalidad conlleva al diálogo y comprensión de las manifestaciones culturales. Promueve la convivencia en el marco de una cultura de paz, la participación comunitaria y la interacción entre culturas. En este contexto, los museos comunitarios son espacios que brindan respeto a la cultura de cada pueblo que tiene expresión en las colecciones resguardadas y contadas a través de la misma comunidad, con arraigo en el pasado.

\section{Conclusiones}

El Museo Comunitario Los Ranchitos es un referente histórico-cultural de la zona mestiza de Nueva Guinea, su contribución abarca la conformación de redes de Museos Comunitarios de Nicaragua y a la memoria e identidad de los pueblos. El trabajo comunitario impulsa el intercambio cultural, contribuyendo al respeto de distintas diversidades culturales.

En la concientización y divulgación ha sido una herramienta para la apropiación de la identidad y la memoria colectiva de los pueblos. Asimismo, es visto como un espacio para el resguardo del patrimonio cultural y la voz viva para la recuperación de la memoria olvidada, a través de la museología presentada en él. 


\section{Agradecimiento}

Esta publicación obtuvo el financiamiento de: NORAD a través del Proyecto RUIICAYHIOA INTERCULTURAL COMMUNICATION LINKAGE PROGRAMME (Número 1300624).

\section{Lista de referencias}

Gamboggi, A. L., \& Melville, G. (S.F.). Museo Comunitario como Técnologia Social en Ámerica Latina.

López, E., \& Ramos, U. (2012). Historia de Nueva Guinea Nicaragua de la pluvioselva a Ciudad Luz . Nueva Guinea, Nicaragua.

López Mairena, E. C., \& Sobalbarro Pao, G. (2016). Memoria: Red de Museos Comunitarios de América. Oaxaca: Red de Museos Comunitrios de América

Martínez, M. (2018). Entrevista Red Nicaraguense Museos Comunitarios.

Morales, T., \& Camarena, C. (2009). Manual para la creacion de museos comunitarios. Bolivia.

Morales, T., Camarena, C., \& Valeriano, C. (1994). Pasos para crear un museo comunitario. México.

Rodrigo, M. (S.F.). La Comunicacion Intercultural.

Ruíz Delgadillo, A., Vizcaya Betancourt, C., \& García Fajardo, J. (Noviembre de 2003). Una Prospección Arqueológica en los Territorios Sur y Sureste del Municipio de Nueva Guinea, Nicaragua. Nueva Guinea, Nicaragua.

Vargas, M. (2018). Entrevistador Red Nicaraguense Museos Comunitarios

Zapata,Yuri. (2007). Historiagrafía, Sociedad y Autonomía. Managua. 